\title{
Ice Navigation Robots and Rovers : A Review
}

\author{
Naresh Kumar K K R ${ }^{1}$, Srikshana Sasidaran², Vijay Anand $S^{3}$, A.Sivaranjani ${ }^{4}$ \\ \{nareshkumarkkr16@gmail.com1, srikshana@gmail.com2,vijayanand2001s@gmail.com3, \\ asr.rae@psgtech.ac..in $\left.{ }^{4}\right\}$ \\ Robotics and Automation Engineering, PSG College of Technology, Coimbatore, India ${ }^{1}$
}

\begin{abstract}
In today's contemporary world, the inquisitiveness to look into and search into the beyond is constantly on the rise. And this snoopy nature is what resulted in the need for over-snow mobility and polar exploration. Since, these environments are hazardous for humans, the demand for snow rovers and glazier robots became quite popular. In this paper, the various contrasting methods employed for making these bots have been analyzed and put to the test.
\end{abstract}

Keywords: Over-snow Mobility, Ice penetrating Bots, Glazier Bots, Ice Rovers, Polar Bots.

\section{Introduction}

The Homo sapiens have always had an unquenchable thirst for knowledge. The curiosity to know what resides in the spaces left unexplored by the human race has led to a lot of technological developments. Be it the less traversed terrains of Mars, or the elusive biogeographic environments of Europa or the tough to navigate polar ice caps of the Arctic and Antarctic continents, snow and intermittent ice and sub-ice poses a common threat.

These conditions are extremely dangerous for human beings to navigate through due to not only the imminent possibilities of natural disasters like avalanches but also even the slightest human error can prove to be extremely fatal.

This led to the need to develop robots and rovers that could navigate not only on ice but also through ice. Besides being used for exploration purposes, these rovers and bots can also be used in observation and espionage grounds.

To facilitate the navigation of the rovers in such tough territories, our scientists and researchers have devised a multitude of methods that have been weighed up in this paper.

\section{Mobility Challenges Presented By Ice And Snow}

\section{Over-snow navigation difficulties:}

One of the major issues, the robots and rovers face while travelling over snow and ice is going immobile when navigating through deep basins. In order for robots to travel over snow, it should compress the snow around it to support its weight, which requires a pressure of about 35 to $50 \mathrm{kPa}$. 
This compression process creates a resistance, which in turn doubles the total resistance faced by the bot. The bot will have reduced endurance as the battery capacity tends to deteriorate with low temperature.

The snow is fathomless when compared to the ground clearance of the bot. Hence the bot or the rover doesn't have any support and sinks to its belly. This increases the motion resistance faced by the bot. Traction is developed in the tracks due to the weight of the belly pan, which results in a rise in the thrust, which in turn results in excessive track slip leading the robot to sink deeper into the snow.

Bots also have trouble executing the steering maneuvers, thus adding to the frictional resistance of the bot and the turning moment requirement escalates. There is a very imminent possibility of snow jamming the track drive mechanism and running gears of the bot.

This will result in the rover ingesting a lot of snow and hinder the motion of the rover. Blizzards and other natural phenomena like avalanches and so on tend to obstruct the vision system of the bot.

\section{Through ice navigation difficulties:}

The bots and rovers need to penetrate a minimum of at least 5 to $10 \mathrm{~cm}$ depth to meet the requirements. But there is the possibility of the thin ice crusts cracking and failing, which would lead the bot to sink to unrecoverable depths.

The ice is a very slippery and hard surface to travel through. It becomes very difficult for the bots to get a grip on and steer, as exerting too much pressure on the crusts might again result in the collapse of the rover.

Since the north and south poles are made up of large blocks of ice that are floating over the ocean, there is a possibility for the ice blocks to collide with each other due to the tides. And when two blocks of ice collide, they tend to form blocks bigger and taller than their predecessors. This will result in the surroundings being even harder for the bot or rover to manipulate through.

Another possible hindrance the ice terrain presents the bot with is the reflectiveness of the ice. The reflective nature of the ice prevents the vision system from functioning with its maximum efficacy.

\section{Methodologies}

Though it is very hard to overcome all these issues and come up with one single integrated solution for all these miscellaneous requirements, our scientists and researchers have come a long way and have come up with a heterogeneous range of methods and rover compositions to get the most out of the available resources and constraints presented with. And all the methods employed to develop a bot or rover with good mobility has been discussed below.

\section{Lightweight rovers:}

Some of the major requirements to facilitate good mobility of the rovers or bots over snow are low ground pressure, a drive system that has a high tolerance towards moisture and snow, and a ground clearance that is high enough relative to the size of the rover. Moreover, most of the times, rovers and bots that have a ground clearance of about 10 centimeters will have the ability to levitate over the snowy terrains.

With these parameters in mind, J.H.Lever and his colleagues [1] have devised and proposed the concept of a light weight unmanned ground vehicle to navigate over the snow 
clad surroundings. The probable solution proposed has been tested by building a bot called 'Snowbot 2'.

The Snowbot 2 has been designed on the basis of the mobility theorem updated by Wong. The very first design parameter put forward by the team is that the bots or rovers should be less than 50 kilograms in weight.

In consideration of the easily compressive nature of the snow, the bots or rovers have to be designed in such a manner that it does not exert a pressure or more than $5 \mathrm{kPa}$ to ensure that the bot does not sink into the snow. The bot or rover should also be planned such that it exercises the pressure equally both across and along its tracks.

To avoid plowing of the bot's belly pan, the bot should have adequate ground clearance. Though this will lead to the necessity of having to compromise on the floatability of the bot over the snow, this characteristic is quite essential to make sure that the bot or rover doesn't ingest snow. Simultaneously, the ground clearance of the rover or the bot should be comparatively higher than the sinkage that the bot might face while navigating through this terrain.

The motors used in the rover should be able to generate sufficient torque to overcome the internal friction developed by the bot while trying to maneuver in the snow. To facilitate the bot to travel over snow, the gross traction must be higher than the resistance developed due to the compression of snow by the bot.

And to make certain that the bot or rover doesn't encounter additional resistance whilst turning in the snow, the turning ratio of the bot should be at least unity or greater than unity. This acts as an indicator of how easily the bot or rover can turn in icy terrains.

The Snowbot 2 is an operator-controlled bot that can be operated remotely within a 100to-150-meter radius and is better suited for short distance functions.

In order to prevent the ingestion of the snow into the rover's drive system, the system has been reengineered with a sprocket drive mechanism to increase the tolerance of the rover or bot towards ice and snow.

Ice penetrating rovers:

Another prospective approach to fulfill the above-mentioned requirements has been developed by NASA primarily to explore the bio-geography of Europa and Mars. The rover has been designed in such a manner that it suits the diverse environments on both Earth and Mars.

The terrain of Mars is still majorly under scrutiny, the only thing known certainly is the presence of overlying ice surfaces and ice deposits with a size range of between 1 meter and 100 meters. Whereas in Europa there are layers upon layers of sub-ice and inter-ice which pose to be an imminent threat to the efficient and hazard free motion of the bot or the rover.

Zimmerman and his teammates [2] have conceived an approach quite similar to the previous one in terms of the structural design aspect. The vehicle is expected to also belong to the light weight category of rovers and should weigh less than 40 kilograms.

The cryobot they have designed to test their hypothesis is called the 'JPL Cryobot'. This bot is significantly smaller in size when compared to its predecessors and weights only around 20 to 25 kilograms.

The proposed system is to use a probe to melt and ice and enter it. The probe used in this bot is 1 meter in length and has a diameter of 15 centimeters. This is also pretty small in size in comparison with the past probes designed for ice penetration purposes.

The probes used in the bot generally need a lot of power to heat and melt the ice. Likewise, the probe used in the JPL bot has a power requirement of 1 kilowatt of thermal 
power. It has a four-section heating system for the passive melting of ice which facilitates simpler steering of the bot.

The bot proposed has an autonomous control system which is facilitated by using an acoustic imaging system for a structured navigation of the bot. This vision system can be used to not only detect the obstacles in the path of the bot but to also send updates on the environment to the operator desk.

The communication system and the sensors are affixed on the top half of the bot to ensure that the communication system remains in pristine condition throughout the mission. Another added advantage of this design is that the communication system also serves the purpose of the anchor when the lower half of the bot penetrates through the ice and travels into the depths to retrieve important bio-geographic data.

In contradiction to the prevailing ice penetration approaches followed by humankind till date which uses unlimited heat to melt the ice, the method proposed here uses only the bare minimal heat to melt the ice, thus reducing the power consumption of the bot.

Apart from being used to melt the ice to penetrate into the ice caps, the probes also help in controlling the direction of the vehicular movement of the bot. The ice which turns into water when melted using the probe also acts as an insulating medium for the rover.

This cryobot uses a two-axis inclinometer to orient the vehicle and has three operational modes to adapt to different types of environments and functional methods. The manual mode is best suited for expeditions on earth and is totally dependent on the operator for instructions.

The passive heating mode is well equipped for melting into the ice layers. The operator has control over the heating probe in particular orientations. In this mode, the path planning system of the cryobot is completely dependent on the inclinometer and the odometer sensors for data.

The last mode being the active water jetting mode can also be termed as the dense ice mode, as it is predominantly used to travel into or through the deep inter-ice layers. This mode allows only the downward movement of the bot to melt ice efficiently in the depths for information and data retrieval.

\section{Large footprint rovers:}

Another approach to master the above mentioned hardships while travelling through glaziers was drawn up by L.Pedersen and his peers [3]. The NOMAD rover originally designed for astro-biological exploration in the Arctic and Antarctic regions in the LORAX mission.

Though this rover was not able to cope up with all the necessary requirements, it is good enough to satisfy the basic necessities in the test conditions.

In contrast to the previous methods coined, this approach advocates an unnaturally larger footprint for the rover in order to support huge payloads and also to have a stable and low center of gravity at the same time.

The NOMAD rover also employs two different vision systems to get the better of the misconceptions in both the systems. The stereo vision system tends to fail due to the monochromatic nature of the images captured by it, making it traversable.

On the other hand, the laser scanning imaging system also faces potential failure at times of snowfall and also due to the reflective characteristic of ice. Hence, both the stereo vision system and the laser scanning imaging system is paired up and used simultaneously.

In order to avoid the probability of the signal clash that will occur because of using two different vision systems, each vision system is tasked with a separate task. The stereo vision system is used to detect the obstacles in the icy terrains, whereas the laser vision system is used more like a cushioning system for the rover and to provide the permissible safeguard. 
The stereo vision system uses a diverse range of algorithms to enable the bot to travel in any given lighting environment. Be it the blindingly bright afternoon lightning or very dark and dull lightning that prevails during snowfall, this vision system facilitates the rover to operate under any lighting conditions.

The NOMAD rover uses a navigation software which houses a variety of control loops and inputs from the servos affixed in the wheels of the rover. These input signals play a major role in providing data for the commanding of the rover and also provides navigational enlightenment to the bot.

To power the rover, the NOMAD rover uses an internal combustion engine and the entities of its locomotion system include a brushless direct current motor, a harmonic drive reducer, an Ackerman steering drive and a spur gear stage. This rover can sustain for 89 days purely based on its inbuilt batteries and fuel systems.

\section{Autonomous rovers:}

Rovers which should be easy to construct and is cost efficient and is also simultaneously capable of operating in the ice sheets of the polar region have been presented. In 3000 USD the total construction of this rover could be done which is $1 / 10$ of the already existing rovers which are used for Polar Regions.

Due to the harsh environment, severe low-power requirements, and economic constraints, the deployment of traditional autonomous rovers in Polar Regions remains difficult.

Polar temperatures often fall below minus 40 degrees Celsius, considerably below the temperature limitations of typical electronics, and wind speeds can easily reach 15 meters per second, causing varying loads on any large profile robotic platform.

More than $10 \mathrm{~km}$ traverses on a single battery charge with the capacity to tow $100 \mathrm{~kg}$ in science payload are required to get good results.

Therefore, Hoffman and his team has developed an economically sustainable alternative. The benefit of the low-cost platform is that the cost could be used to instrument the rover or expand scope and logistical support. It can also be sent to more hazardous field as even if it gets damaged or any part of the rover get damaged it can be easily 3D printed or can be replaced cheaply.

\section{Path Planning For Unhindered Mobility}

Most of the rovers and bots developed to facilitate movement and mobility in the Polar Regions, snow clad terrains and glaziers are designed to be completely autonomous functioning vehicles.

One of the most important problems to be solved while designing these autonomous vehicles is path planning. The task of the path planning is to have collision free path, shortest and smooth path. The navigation strategy can be divided into two namely, global navigation and local navigation based on the environment.

Global navigation generates the collision free path for the rover based on the current and past perceptive environment and on a known environmental map. Global path planning algorithms are useful in path finding in static environment and requires prior map information, when the complexity and uncertainty of the environment increases the efficiency of these algorithm will be greatly reduced. 
And also, in the scenario of when the robot encounters the unseen obstacles blocking the pre-planned path, re-planning of the path should be done based on the current environment, this will increase computational process and response will deteriorate.

The local path planning does not require much information or no prior information of the environment. It reacts to the obstacles and changes its direction to avoid the obstacles. This type of path planning is suitable for dynamic environment. Hence, this method is preferred to be incorporated in the snow-clad environment and icy layers.

The traditional path planning algorithm depends heavily on the surrounding environment and lacks flexibility and adaptability where the modern robots require in dynamic environment. To overcome deep reinforcement learning has been proposed. The ability of the deep learning like interpreting and decision-making are integrated in the deep reinforcement learning.

Discussed below is some of the better suited path planning algorithms for constantly varying environments. These algorithms tend to make the bots and rovers make better navigation and mobility decisions when functioning autonomously without any form of supervision in remote and hazardous environments.

\section{Actor critic method:}

Actor critic combines the best part of the Value-iteration methods and Policy-gradient methods. It predicts both optimal policy function and value function. The agent uses the value function (Critic) value to update the optimal policy function(actor) so that the optimal policy function output finer actions that results in higher reward.

\section{$Q$ Learning algorithm:}

Q-learning algorithm is one of the Reinforcement learning algorithms; it provides the ability to avoid obstacles which are dynamic in nature. It works based on the value function estimation. It is also known as Q table algorithm. In the process of this algorithm, the agents select the best action by repeated interaction with the environment in trial-and-error manner. The correct Q value increases the reward, whereas wrong action will decrease the reward. By repeating the actions in different states and updating the $Q$ value, the algorithm selects the optimal action to learn the optimal behavior strategy. This algorithm can avoid obstacles effectively. The main disadvantage of Q-learning is that it requires lots of memory for the Q table.

Deep $Q$ Network:

DQN is based on the Q learning algorithm with added Neural network to approximate the value function, input action and state, output value, and using good parameters to train the Neural Network. Then the input is provided to get corresponding output.

There is one main problem using neural networks to estimate Q-value: due to the nonlinearity of the deep neural networks, it causes the Q learning algorithm to not converge and become unstable during the learning. Commonly, the Deep Q Network and empirical playback mechanism combined with the target Network is used to solve this above-mentioned problem. The term Deep Q Network with the target Network is adding a same structure of neural network to the original neural network. The Neural network before adding the additional network (original neural network) is called evaluation network and the original neural network added with the above-mentioned structure is called Target network. The evaluation network gets updated, after sometime the numbers of updates are finished, and then the Target network gets updated, to lower the correlation between calculating the target $\mathrm{Q}$ value and updating the $\mathrm{Q}$ network parameters this happens during learning process. After the completion of Neural Network training, the network parameters are relatively fixed and corresponding function value does not change. 
DQN can achieve human level performance in many Atari video games by estimating action of an agent. However, it can handle complex observation spaces; it can only be used discrete action spaces. So DQN cannot be applied in continuous action spaces, we need to use another algorithm that can solve this problem. Another disadvantage of this algorithm is its slow convergence speed and excessive randomness.

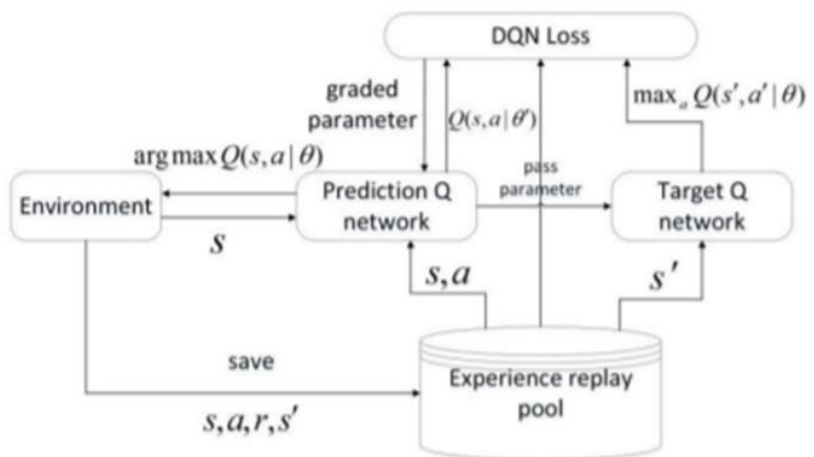

Figure 1. DQN with target network

\section{Deep Deterministic Policy Gradient:}

Deep deterministic policy gradient is the combination of DQN and Actor critic algorithm. It is the fusion of neural network and reinforcement learning. It uses actor critic algorithm to learn continuous action state policies. It uses neural network for each of the actor network and critic network.

These Networks computes the action prediction for the current state and for each time step the temporal difference error signal is generated. The current state is the input to actor network, the output is the action chosen for a continuous action space in the form of a real value. Exploration is the biggest challenge in the continuous space action. To encounter this exploration policy $\mu^{\prime}$ need to be constructed by adding a noise sampled from a noise process to the actor network.

To generate temporally correlated exploration efficiency in physical control problems, Ornstein-Uhlenbeck process is most widely used, recently results showed that mean-zero Gaussian noise can work perfectly well.

DDPG uses replay memory to store the state, actions, rewards and the new state that the agent explored during the training. The intelligent agent allowed learning from recent memories from the experience replay, which increases the learning speed and breaking the temporal correlations. It is possible to see substantial improvement in the agent performance even with a short memory. The advantage of this algorithm is that it can be used in continuous space action and it finds the path in the shortest time when compared to the above-mentioned algorithms. 


\section{Discussions}

Despite the various methods being devised to overcome the hazards presented by the icy terrains and the snow-clad regions, each of the proposed approaches have their own sets of drawbacks and advantages when it comes to adapting and meeting the requirements of such a demanding environment.

When it comes to the initially suggested approach of Snowbot 2, the rover can still be immobilized if it is driven into places and depressions near bushes and trees, as the snow depositions in these regions are comparatively higher when compared to the other regions.

And since this rover doesn't have any inbuilt vision systems, this bot is highly dependent on the instructions it receives from the operator to decide on its next movement. As it also doesn't have any means to provide visual feedback to the operator, it is essential that the operator of the bot remains in close proximity of the bot. The operator has to stay within a 100 meter to 150 -meter radius of the rover.

Another major drawback is that, the Snowbot 2 doesn't allow any backward or reverse motion. This feature of the bot proves to be highly problematic because in cases where the bots get stuck in small holes and bumps, the only scope of recovery is going backwards. But since this bot forbids any kind of reverse motion, the possibility of recover becomes almost negligible.

But on the other hand, these rovers have a very high tolerance to snow, thanks to the sprocket drive system introduced. Due to this feature, even if a good amount of snow is ingested by the bot, it can still continue to move in the glaziers.

One of the key advantages of the Snowbot 2 is that, it is very light in weight when compared to the other rovers and weighs only around 20 kilograms. This enables the bot to navigate and move through the glaziers and ice crusts, which usually tend to break when travelled on foot.

Apart from these, this rover exerts a very minimalistic amount of ground pressure and has a greater ground clearance. This is one of the major reasons which stop the bot from consuming snow.

Above all, the Snowbot 2 has a humongous structural integrity which enables the rover to traverse to a variety of regions including frozen marshes, rivers and lakes.

When it comes to the JPL Cryobot, it is one of the most structurally sound and accurate models when it comes to meeting the fluid dynamics and heat transfer requirements to navigate through the sub-ice and inter-ice environment.

It has also performed exceptionally well under a suitable test environment and could effectively melt ice at a rate of about 0.5 to 1 meter per hour. And despite other minor drawbacks, it satisfies the other requirements when it comes in terms of power, vehicle size and functionality.

The double heater, split nose configuration of the heating probe of the cryobot proves to be an added advantage for the system. In the initial version of the JPL Cryobot, water has been used as the primary insulating agent and more importance has been given to the usage of the water jet vortices to remove the debris and melt them.

But there is scope for further improvement in this bot in terms of the steering dynamics. Rigorous modeling and testing is also required to improve upon the existing vision systems. And more research is needed to be put into action to ensure the betterment of the existing instruments used and the communications systems to suit even deeper descent applications. 
Now, when it comes to the NOMAD rover, it is one of the heaviest rovers ever developed to maneuver through snow and ice and weighs over 700 kilograms and is also massive in its size. This results in limiting the environments in which it can navigate through, as the weight of the rover might cause the thin ice crusts and glazier layers to break down, which will ultimately lead the rover to sink into unrecoverable conditions.

Since the NOMAD uses an internal combustion engine, it needs a constant supply of fuel. Practically, it is very difficult and also proves to be an added burden to the bot if it needs to constantly carry fuel when going on longer expeditions. But if the power requirement of the bot is reduced, solar energy and wind energy can be used as other feasible alternatives for the internal combustion engines.

But since the rover uses a harmonic drive, it has a very high internal resistance and is totally inefficient at no load conditions. This bot needs a minimum of 75 watts to drive at a speed of 0.5 meters per second.

Hence in order to minimize the power consumption, from 437 to 222 watts, the drive motors can be supplanted with motors having a larger diameter with multiple poles. The waste heat generated by the rover can be used to heat the main electronics box to ensure that it remains within the working temperature conditions.

\section{References}

[1] J.H.Lever, S.A.Shoop, R.I.Bernhard, "Design of lightweight bots for oversnow mobility", Journal of Terramechanics, 2009.

[2] Wayne Zimmerman, Robert Bonitz, Jason Feldman, "Cryobot: An ice penetrating robotic vehicle for Mars and Europa”, IEEE, 2001.

[3] Liam Pendersen, David Wettergreen, Dimi Apostolopoulos, Chris McKay, Matthew DiGoia, Dominic Jonak, Stuart Heys, James Teza, Michael Wagner, IEEE

[4] Andrew O. Hoffman, Hans Christian Steen-Larsen, Knut Christianson, Christine Hvidberg, “ A low cost autonomous rover for polar science", Geoscientific Instrumentation Methods and Data Systems, 2019.

[5] Yu J, Su Y, Liao Y,"The path planning of mobile robots by neural networks and hierarchial reinforcement learning",Front, Neurobot, 14:63.

[6] J.Xin, H.Zhao, D.Liu, M.Li, "Application of deep reinforcement learning in mobile robot path planning", 2017 Chinese Automation Congress(CAC), 2017.

[7] Wang, Xiaoqi \& Jin, Lina \& Wei, Haiping, " The shortest path planning based on reinforcement learning", Journal of physics: Conference Series

[8] J.C.Jesus, J.A.Bottega, M.A.S.L.Cuadros, D.F.T.Gamarra, "Deep deterministic policy of gradient for navigation of mobile robotics in simulated environments", 19th International Conference on Advanced Robotics (ICAR), 2019.

[9] Karur.K, Sharma.N, Dharmatti.C, Siegel.J, “A survey of path planning algortihms for mobile robots", Vehickes, 2021.

[10] De Jesus, J.Kich, V.A.Kolling, A.H. et al., "Soft actor-critic for navigation of mobile robots", J Intell Robot Syst, 2021. 